\title{
\begin{tabular}{l|l} 
Mitraries & DSpace@MIT
\end{tabular}
}

\author{
MIT Open Access Articles
}

\section{Reduced-Order Cue-Signal-Response Modeling for Angiogenic Cell Migration Control: A Principal Signal Approach}

The MIT Faculty has made this article openly available. Please share how this access benefits you. Your story matters.

Citation: Asada, H. Harry. “Reduced-Order Cue-Signal-Response Modeling for Angiogenic Cell Migration Control: A Principal Signal Approach.” ASME 2010 Dynamic Systems and Control Conference, Volume 1, 12-15 September, 2010, ASME, Cambridge, Massachusetts, 2010, pp. 445-51. (C) 2010 by ASME

As Published: http://dx.doi.org/10.1115/DSCC2010-4246

Publisher: ASME International

Persistent URL: http://hdl.handle.net/1721.1/118796

Version: Final published version: final published article, as it appeared in a journal, conference proceedings, or other formally published context

Terms of Use: Article is made available in accordance with the publisher's policy and may be subject to US copyright law. Please refer to the publisher's site for terms of use. 


\title{
DSCC2010-१०००
}

\section{REDUCED-ORDER CUE-SIGNAL-RESPONSE MODELING FOR ANGIOGENIC CELL MIGRATION CONTROL: A PRINCIPAL SIGNAL APPROACH}

\author{
H. Harry Asada \\ Department of Mechanical Engineering \\ Massachusetts Institute of Technology \\ Cambridge, Massachusetts 02139 \\ Email: asada@mit.edu
}

\begin{abstract}
A cell's behavior in response to stimuli is governed by a signaling network, called cue-signal-response. Endothelial Cells (ECs), for example, migrate towards the source of chemoattractants by detecting cues (chemo-attractants and their concentration gradient), feeding them into an intra-cellular signaling network (coded internal state), and producing a response (migration). It is known that the cue-signal-response process is a nonlinear, dynamical system with high dimensionality and stochasticity. This paper presents a system dynamics approach to modeling the cue-signal-response process for the purpose of manipulating and guiding the cell behavior through feedback control. A Hammerstein type model is constructed by representing the entire process in two stages. One is the cue-to-signal process represented as a nonlinear feedforward map, and the other is the signal-to-response process as a stochastic linear dynamical system, which contains feedback loops and auto-regressive dynamics. Analysis of the signaling space based on Singular-Value Decomposition yields a set of reduced order synthetic signals, which are used as inputs to the dynamical system. A predictionerror method is used for identifying the model from experimental data, and an optimal system order is determined based on Akaike's Information Criterion. The resultant low order model is capable of predicting the expected response to cues, and is directly usable for feedback control. The method is applied to an in vitro angiogenic process using microfluidic devices.
\end{abstract}

\section{INTRODUCTION}

Cell behavioral functions, such as migration, proliferation, and death, are governed to a large extent by networks of signaling proteins whose activities are modulated by a variety of extracellular cues [1]. Those cues are environmental conditions and agents, including chemical ligands, mechanical forces, pathogens and so forth. Cells detect those cues with receptors and feed them into their signal transduction pathways, which result in cell decisions and actions. This cue-signalresponse process is the key to understanding physiological cell behaviors. In the past few decades, investigations into signaling networks have been active in molecular cell biology, and many signaling pathways and associated proteins have been identified. Yet, their collective, quantitative effects are still poorly understood [2].

The objective of this work is to fill the gap between the biological model delineated in the signaling network and the quantitative dynamic model necessary for simulation and control in the engineering arena. Recent progress in microfluidic technology [3] opens up the possibility to guide and manipulate cell behaviors in tightly controlled in vitro environment. Yet, the lack of a quantitative dynamic model that can reliably predict cell behaviors hinders the development of effective controls. Such a quantitative model usable for control must be low dimensional and identifiable from experimental data.

In the systems biology and computational biology literature, multivariate data analysis has been used extensively for mining DNA microarray datasets [4,5]. Data analysis of signaling networks has also been performed by several groups. Among others, the Lauffenburger group at MIT has pioneered the cue-signal-response analysis based on Partial Least Squares (PLS) estimate [6]. A particular combination of signals has been successfully identified based on PLS data analysis that highly pertains to programmed cell death. The identified signals are low dimensional, yet informative in predicting the cell's response to cues. 
Cue-signal-response processes are highly nonlinear and dynamic. Furthermore, biological data in general have a high level of variability and uncertainties. It is a challenge to obtain a control-oriented model from statistics and multivariate data analysis alone. Dynamical systems methodologies, such as system identification, stochastic estimation, and information theoretic analysis, will be powerful tools when combined with statistical analysis. This paper presents a systematic modeling and identification method for obtaining a control-oriented, loworder model from signaling network and experimental data. The basic model structure is Hammerstein model, consisting of a nonlinear algebraic map cascaded to a linear dynamic process. Using Singular-Value Decomposition and Akaike's Information Criterion, an optimal, low-dimensional, nonlinear dynamic model will be derived in a systematic manner.

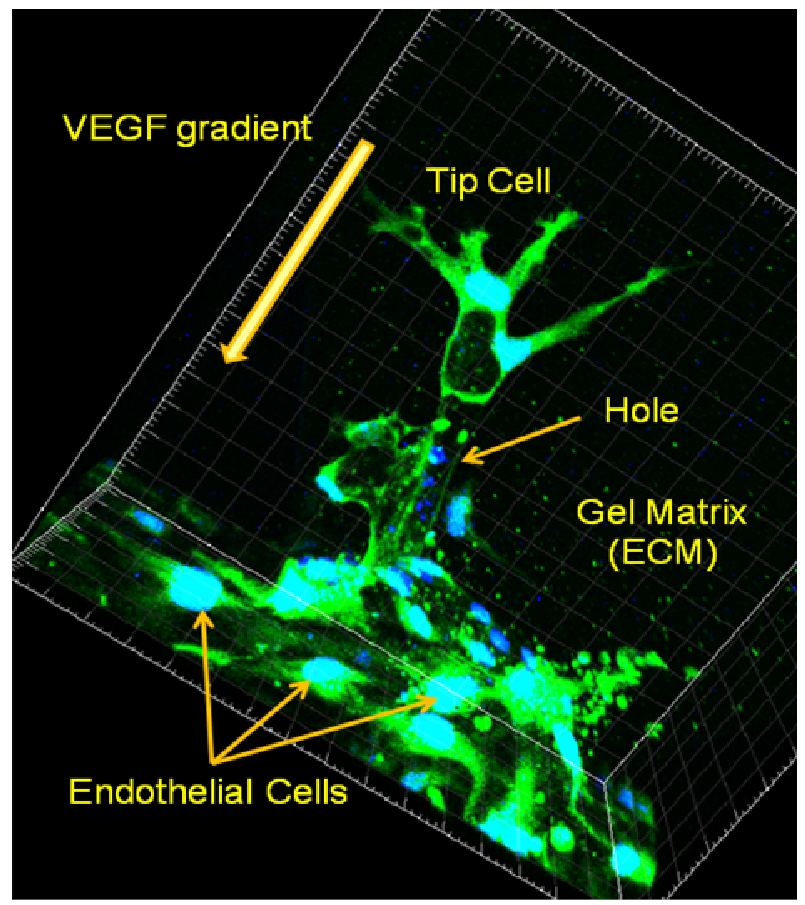

FIGURE 1. CONFOCAL MICROSCOPE IMAGE OF ENDOTHELIAL CELL SPROUTING EXPERIMENT USING IN VITRO MICROFLUIDIC CULTURE.

This modeling methodology, although applicable to various biological systems, will be developed in the context of Endothelial Cell migration in angiogenesis microfluidic cell cultures. What will follow are a brief introduction to angiogenesis cell migration, Hammerstein modeling framework, nonlinear modeling of cue-to-signal processes, signaling space reduction, dynamic modeling and identification of signal-to-response process, and application of the methodology to angiogenic migration speed control.

\section{ANGIOGENIC CELL MIGRATION}

Angiogenesis is one process of generating a vascular network. Since tissues cannot survive without blood supply and waste removal, angiogenesis is critically important in broad medicine and life science fields, including regenerative medicine, cancer treatment, drug development, and wound healing. As shown in Fig. 1, Endothelial Cells (ECs) residing in a blood vessel sprout out in response to various growth factors, such as Vascular Endothelial Growth Factor (VEGF), Angiopoietin (Ang) I and II, basic Fibroblast Growth Factor (bFGF), and Angiostatin. Those growth factors are secreted when tissues need more oxygen (are hypoxic) or become wounded. Cancerous tumors demand blood supply by secreting these growth factors and causing sprouts to extend toward the tumor. As shown in the figure, a leading cell, called a tip cell, detects the gradient of growth factor concentration, in particular, VEGF gradient, and migrates towards higher concentration [7]. When migrating, a tip cell secrets a protease, termed Matrix Metalloproteinase (MMP), to degrade the surrounding gel matrix. As a result, a hole is created in the Extra-Cellular Matrix (ECM) after a tip cell goes through the gel matrix.

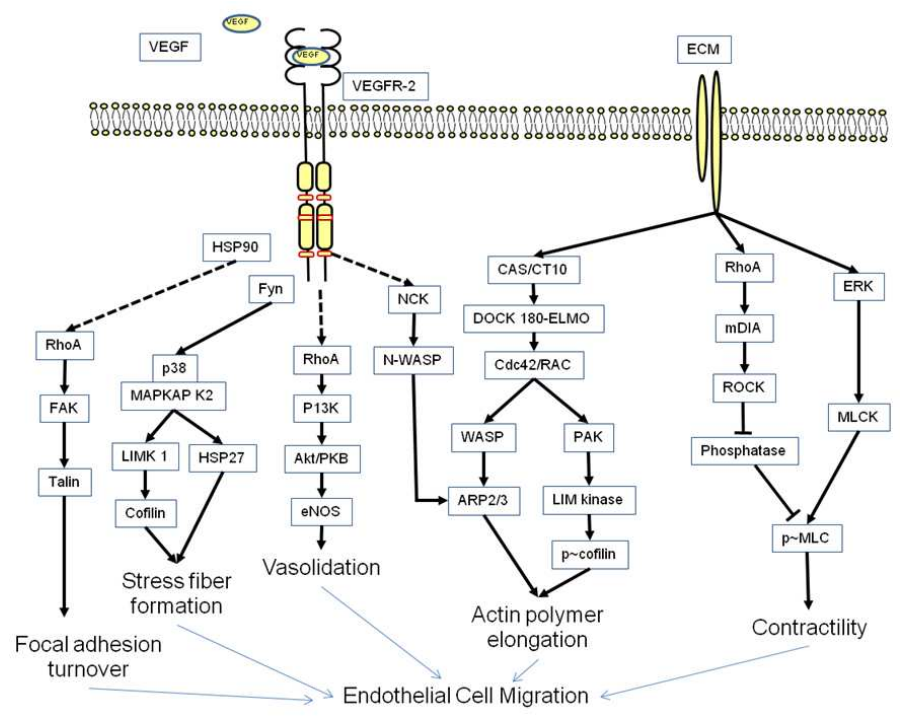

FIGURE 2. SIMPLIFIED INTRACELLULAR SIGNALING NETWORK. ADOPTED FROM $[1,8,9]$.

Each tip cell is followed by "stalk cells," which construct a tubular structure. Stalk cells crawl along the wall of the hole created by the tip cell. A group of stalk cells spread out across the wall and create a tubular structure by covering the wall surface. When a tip cell moves too fast, it does not properly degrade the surrounding collagen gel, resulting in a narrow hole. The author's research group has observed in microfluidic experiments that stalk cells are unable to follow a tip cell when the tip cell migration speed is too fast, leaving only a narrow hole. On the other hand, when the tip cell speed is too slow, stalk cells get stuck and form a lump, rather than extending a blood vessel. The tip cell migration speed must be regulated, so that a functional blood vessel can be created.

The tip cell behavior is governed by a cue-signalresponse network. Figure 2 shows a simplified version of 
signaling network with receptors of VEGF and special transmembrane adhesion receptors, called Integrins, which bind to ECM. In response to these cues, the tip cell adheres to ECM and generate a traction force for migration.

The actual Endothelial Cell has more complex signaling networks, generating diverse functional responses, including proliferation, apoptosis, and quiescence [1,8,9]. This paper focuses on the dynamic behavior of tip cell migration, the regulation of which plays a key role in forming a proper vasculature, as described above.

\section{HAMMERSTEIN MODELLING FRAMEWORK}

As shown in the above diagram, the cue-signal-response process is highly nonlinear and dynamic. Signaling pathways involve a number of nonlinear nodes due to multiplicative nature of signal transduction, signal saturation, etc. Diffusion of signaling molecules, binding to receptors, etc. are dynamical processes with slow transient response. One way of modeling such complex systems with prominent nonlinearity and dynamics is to separate the process between the one consisting of nonlinear, but all forward path transmissions and the one having feedback loops or auto-regression. The Hammerstein model is a modeling framework representing a nonlinear dynamical system as a cascaded process of nonlinear algebraic map followed by a linear dynamical process. Separation between nonlinear forward path dynamics and linear autoregressive dynamics brings about mathematical tractability, allowing us to apply some powerful tools and methodologies developed in the system dynamics and control discipline.

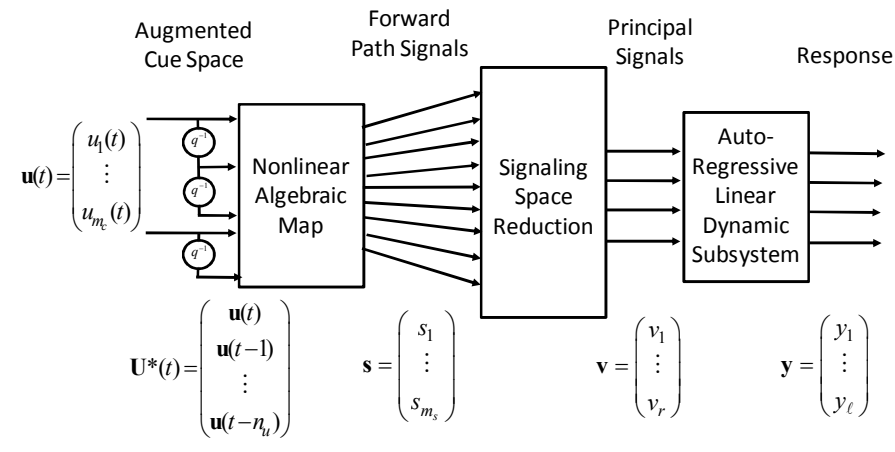

FIGURE 3. EXTENDED HAMMERSTEIN MODEL.

Figure 3 shows a block diagram of the Hammerstein model. Cues are collectively represented as an input vector, $\mathbf{u}=\left[u_{1} \cdots u_{m_{c}}\right]^{T} \in \mathfrak{R}^{m_{c} \times 1}$, which is mapped to a highdimensional vector, $\mathbf{s}=\left[s_{1} \cdots s_{m_{s}}\right]^{T} \in \mathfrak{R}^{m_{s} \times 1}$, termed a forward path signal vector. The relationship between input cues and these signals is nonlinear, but is assumed to be free of feedback loops. Along the pathways from cues to forward path signals some time delays may be present in transmitting signaling molecules. Many of the signal transductions involved in Fig. 2 are cascaded ones, which can be treated as a series of delayed signal transmissions. Feedback loops and auto-regressive nature of dynamics are modeled separately in the downstream linear dynamic process. This feed-forward vs. feedback separation makes the modeling and identification amenable.

As reported in the literature, the signaling space is redundant in the sense that a small number of principal components can approximate the signaling space with reasonable accuracy [6]. Let $\mathbf{v}=\left[v_{1} \cdots v_{r}\right]^{T} \in \mathfrak{R}^{r \times 1}$ be an $r$ dimensional reduced signaling space, where $r \ll m_{s}$ and $v_{i}$ is the coordinate of the $i$-th largest principal component. These variables are termed principal signals. In response to the principal signals a set of output response variables, $\mathbf{y}=\left[y_{1} \cdots y_{\ell}\right]^{T} \in \mathfrak{R}^{\ell \times 1}$, are produced. The relationship between principal signals and output responses is dynamic and autoregressive, but is assumed to be linear.

The central problem in this paper is to identify the nonlinear dynamic process from cues $\mathbf{u}$ to response $\mathbf{y}$, based on a dataset consisting of $N$ pairs of cue-response data:

$$
\mathbb{Z}=\{(\mathbf{u}(t), \mathbf{y}(t)) \mid t=1, \cdots, N\}
$$

The dynamic model relating response $\mathbf{y}(t)$ to a time sequence of cues $\mathbf{u}(t)$ must predict the response with minimum mean error and small error covariance. This requires an effective model structure that represents the expected response in a compact form containing least parameters to identify.

The identification process may be divided into three steps:

- The first step is to generate a nonlinear map from cues $\mathbf{u}$ to forward path signals $\mathbf{s}$, based on known signaling network and available data.

- The second step is to reduce the high-dimensional, signaling space to a compact principal signal space of $\mathbf{v}$.

- The final step is to generate a linear auto-regressive dynamic model relating response $\mathbf{y}$ to principal signals $\mathbf{v}$ with minimal complexity.

These steps will be described in the following sections.

\section{CONSTRUCTING A REDUCED-ORDER NONLINEAR MAP FROM CUES TO PRINCIPAL SIGNALS}

Signal transduction pathways are complex, containing a number of nonlinear transductions of signaling molecules. Each node on a pathway is associated with specific protein production, protein-to-protein interactions, protein enzymatic activities, and others. Protein-to-protein interactions pertain to multiplicative operations, e.g. $g_{1}=u_{1}(t) \times u_{2}(t)$, where $u_{1}(t)$ and $u_{2}(t)$ are intensity of cues 1 and 2, respectively. Protein enzymatic activities can be represented as gate operations, e.g. $g_{2}=u_{1}(t) \times \Gamma\left(u_{2}(t) ; \underline{u}\right)$, where $\Gamma(u ; \underline{u})$ is a threshold function with threshold level $\underline{u}$ :

$$
\Gamma(u ; \underline{u})= \begin{cases}0 ; & u \leq \underline{u} \\ 1 ; & u>\underline{u}\end{cases}
$$


With this threshold function, the nonlinear function $g_{2}$ represents that the output from the enzymatic node is generated from $u_{1}(t)$ only when $u_{2}(t)$ exists with an intensity higher than the threshold level.

These activities at the network nodes inevitably saturate, and the output sensitivity to input often decreases as the input increases too far. Saturation functions, such as sigmoid functions, can be used to model this behavior.

Furthermore, signaling molecules, when reaching a node on a pathway, may have some time delay due to diffusion and binding dynamics coupled with threshold and saturation nonlinearity. Multiplicative terms may include $g_{3}=u_{1}(t-1) \times u_{2}(t), g_{4}=u_{1}(t-1) \times u_{2}(t-3)$, etc., which are functions of delayed terms.

To represent these, we first augment the input cue space by adding discrete-time delayed signals:

$$
\mathbf{U}^{*}(t)=\left[\mathbf{u}(t)^{T}, \mathbf{u}(t-1)^{T}, \cdots \mathbf{u}\left(t-n_{U}\right)^{T}\right]^{T} \in \mathfrak{R}^{m_{c}\left(n_{u}+1\right) \times 1}
$$

and create a group of nonlinear functions:

$$
\left\{g_{j}\left(\mathbf{U}^{*}\right) \mid j=1, \cdots, m_{g}\right\}
$$

including multiplicative, saturation, threshold, and other nonlinear functions, which are likely to be involved in the signaling network. We represent the forward path signals as linear combinations of these building block nonlinear functions:

$$
s_{i}=\sum_{j=1}^{m_{g}} w_{i j} g_{j}\left(\mathbf{U}^{*}\right), \quad i=1, \cdots, m_{s}
$$

Note that the above equation represents nonlinear algebraic functions mapped from the augmented input cue space with no auto-regression and feedback.

In molecular biology, intracellular signals have been measured with various techniques, including Western blots, kinase assays, and imaging [6]. These data can be used to establish the functional relationship in the form of Eq. (4). To this end the experimentally measurable signals are collectively represented as

$$
\mathbf{S}_{e x}=\left[s_{1}, \cdots, s_{m_{e}}\right]^{T} \in \mathfrak{R}^{m_{e} \times 1}
$$

Based on Eq.(4), these experimentally measurable signals can be related to augmented input cues and nonlinear functions:

$$
\mathbf{S}_{e x}\left(\mathbf{U}^{*}\right)=\mathbf{W} \cdot \mathbf{g}\left(\mathbf{U}^{*}\right)
$$

where $\mathbf{W}$ is a matrix of weights $w_{i j} ; \mathbf{W}=\left\{w_{i j}\right\} \in \Re^{m_{e} \times m_{g}}$, and $\mathbf{g}\left(\mathbf{U}^{*}\right)=\left[g_{1}\left(\mathbf{U}^{*}\right), \cdots, g_{m_{g}}\left(\mathbf{U}^{*}\right)\right]^{T}$. Given experimental data: $\left\{\left(\mathbf{U}^{* k}, \mathbf{S}_{e x}^{k}\right) \mid k=1, \cdots, N_{s}\right\}$, the least square estimate of the parameter matrix is obtained:

$$
\hat{\mathbf{W}}^{L S}=\Psi \Phi^{T}\left(\Phi \Phi^{T}\right)^{-T}
$$

where

$$
\Phi=\left[\mathbf{g}\left(\mathbf{U}^{* 1}\right), \cdots, \mathbf{g}\left(\mathbf{U}^{*} N_{s}\right)\right] \in \mathfrak{R}^{m_{g} \times N_{s}}
$$

is assumed to be of full rank, $m_{g} \leq N_{s}$, and

$$
\Psi=\left[\mathbf{S}_{e x}\left(\mathbf{U}^{* 1}\right), \cdots, \mathbf{S}_{e x}\left(\mathbf{U}^{* N_{s}}\right)\right] \in \mathfrak{R}^{m_{e} \times N_{s}}
$$

Experimentally measurable signals are often a small fraction of the whole signals in a complete signaling network. It is known that the signaling space is high dimensional and redundant. Therefore, it is possible to reduce the signaling space by finding significant components that span the space. Applying Singular-Value decomposition to the least-square weighting matrix yields:

$$
\hat{\mathbf{W}}^{L S}=\mathbf{V}_{s} \mathbf{D} \mathbf{V}_{g}^{T}
$$

where $\mathbf{V}_{s} \in \mathfrak{R}^{m_{e} \times m_{e}}$ and $\mathbf{V}_{g} \in \mathfrak{R}^{m_{g} \times m_{g}}$ are unitary matrices, and $\mathbf{D} \in \mathfrak{R}^{m_{e} \times m_{g}}$ is a matrix containing singular values of $\hat{\mathbf{W}}^{L S}$ in its diagonal elements with 0 elsewhere.

$$
\mathbf{D}=\left(\begin{array}{ccccc}
\lambda_{1} & 0 & \cdots & \cdots & 0 \\
0 & \lambda_{2} & & & \vdots \\
\vdots & & \ddots & & \vdots \\
0 & \cdots & \cdots & \lambda_{m_{e}} & 0
\end{array}\right)
$$

where the singular values are placed in descending order: $\lambda_{1} \geq \lambda_{2} \geq \cdots \geq \lambda_{m_{e}}$. In literature [6], the signaling space of human colon carcinoma cells is dominated by the first few singular values covering $80 \sim 90 \%$ of the input-output relationship. Therefore, we consider truncating the above singular value matrix $\mathbf{D}$ to a lower dimensional matrix $\mathbf{D}_{0} \in \mathfrak{R}^{r \times r}$ by taking only the $r$ by $r$ top-left corner matrix:

$$
\mathbf{D}=\left(\begin{array}{ccccc}
\mathbf{D}_{0} & 0 & \cdots & \cdots & 0 \\
0 & \lambda_{r+1} & & & \vdots \\
\vdots & & \ddots & & \vdots \\
0 & \cdots & \cdots & \lambda_{m_{e}} & 0
\end{array}\right)
$$

and define a set of new variables by truncating $\mathbf{V}_{g} \in \mathfrak{R}^{m_{g} \times m_{g}}$ as

$$
\mathbf{v}\left(\mathbf{U}^{*}\right)=\mathbf{D}_{0} \mathbf{V}_{g 0}^{T} \mathbf{g}\left(\mathbf{U}^{*}\right)
$$

where $\mathbf{V}_{g 0}^{T} \in \mathfrak{R}^{r \times m_{g}}$ consists of the first $r$ rows of the unitary matrix $\mathbf{V}_{g}^{T}$, each row vector of which points in the direction of the eigen vector in the $m_{g}$ dimensional vector space associated with the singular value $\lambda_{i}, i=1 \cdots r$.

The physical meaning of the new synthetic signals is a compressed set of signals containing most information that is coded from input cues. The validity of these compressed variables is assured only for the experimentally measurable 
signals. However, considering the redundant nature of signaling space and assuming that most of significant signaling activities are reflected in the experimentally measurable signals, the new variables represent the core information from which various signals, both measurable and un-measurable, can be generated. We call these new variables Principal Signals. Note that the number of principal signals is no larger than that of experimentally measureable signals.

\section{IDENTIFYING THE DYNAMIC PROCESS FROM PRINCIPAL SIGNALS TO RESPONSE}

\section{Stochastic Linear Dynamic Model and Predictor}

Treating the principal signals, $\mathbf{v}=\left[v_{1}, \cdots, v_{r}\right]^{T}$, as inputs, the dynamic process from $\mathbf{v}$ to output response $\mathbf{y}$ is now considered in this section. Note that the nonlinear process from the cue space to the signaling space described as algebraic functions is basically a process of unilateral, forward path transmissions, having no feedback or auto-regression. In contrast, the dynamic process leading to output response, e.g. cell migration speed, contains bidirectional physical interactions, which cannot be modeled as unilateral transmission. Viscous damping between the tail of a migrating cell and ECM, for example, is an auto-regressive dynamics. These dynamics will be modeled in this section and its model identification will be discussed in the following section.

We assume that the process is a linear, time-invariant system described by

$$
\mathbf{y}(t)=\mathbf{G}(q) \mathbf{v}(t)
$$

where $\mathbf{G}(q)$ is a $\ell$ by $r$ discrete-time, transfer matrix with time shift operator $q$. The transfer matrix is assumed to be BIBOstable. The problem is to identify the transfer matrix from the dataset given by Eq. (1). Since $\mathbf{v}(t)$ can be obtained from cues $\mathbf{u}(t)$ based on the nonlinear map of Eq. (12), the dataset can be converted to

$$
\mathbb{Z}_{v}=\left\{\left[\mathbf{v}\left(\mathbf{U}^{*}(t)\right), \mathbf{y}(t)\right] \mid t=1, \cdots, N\right\}
$$

An important aspect in modeling dynamics of biological systems is a high degree of stochasticity and heterogeneity. A cell's response, although exposed to the identical cues, differs every time the experiment is repeated. It is important to manifest the stochastic nature of cue-signal-response process and identify the properties of the randomness. It would be effective if process noise and measurement noise were identified separately, as in the case of Kalman filter. In reality, however, it is often infeasible to identify them separately. Instead, we consider a combined random process that is observed at the output:

$$
\mathbf{y}(t)=\mathbf{G}(q) \mathbf{v}(t)+\mathbf{H}(q) \mathbf{e}(t)
$$

where $\mathbf{H}(q)$ is a $\ell$ by $\ell$ transfer matrix, which is $\mathrm{BIBO}$ inversely-stable and monic, and $\mathbf{e}(t)$ is a $\ell$-dimensional uncorrelated, zero mean values, random noise vector. Now the problem is to identify both $\mathbf{G}(q)$ and $\mathbf{H}(q)$ from the dataset (14).

It should be noted that the stochastic transfer function form in Eq. (15) is convenient for system identification as well as for a class of control design. The one-step ahead predictor of output response can be derived directly from Eq. (15). Given observations $\mathbf{y}(1), \mathbf{y}(2), \ldots, \mathbf{y}(t-1)$ and an input sequence, the maximum a posteriori estimate of output $\mathbf{y}(t)$ is given by the following expression [10]:

$$
\hat{\mathbf{y}}(t \mid t-1)=\mathbf{H}(q)^{-1} \mathbf{G}(q) \mathbf{v}(t)+\left(\mathbf{I}-\mathbf{H}(q)^{-1}\right) \mathbf{y}(t)
$$

Note that, since $\mathbf{H}(q)$ is monic, the second term on the right hand side does not include $\mathbf{y}(t)$, but is a function of $\mathbf{y}(t-1), \mathbf{y}(t$ 2), ...

Repeatedly using the above one-step-ahead predictor, an optimal predictor of $k$-step-ahead output, $\hat{\mathbf{y}}(t+k-1 \mid t-1)$ can be obtained as well. Preview control and other prediction-based controls can be designed directly from this predictor. Since cell migration is a slow process having a long latency time, such prediction-based controls will be useful. For state feedback, the predictor of Eq. (16) can also be used as a state estimator. Combining output observations and the prediction, one can estimate state variables under mild conditions. Once the state is estimated, closed loop control around the state can be constructed.

\section{Prediction-Error Based System Identification}

Consider parametric system identification for $\mathbf{G}(q)$ and $\mathbf{H}(q)$. Suppose that the two transfer functions include totally $d$ parameters to identify. Collectively they are denoted by parameter vector $\theta \in \mathfrak{R}^{d \times 1}$. From the one-stepahead predictor, Eq.(16), output prediction error $\Delta \mathbf{y}\left(t \mid t-1 ; \theta, \mathbf{U}^{*}(t)\right)$ is given by

$$
\begin{array}{r}
\Delta \mathbf{y}\left(t \mid t-1 ; \theta, \mathbf{U}^{*}(t)\right)=\hat{\mathbf{y}}\left(t \mid t-1 ; \theta, \mathbf{U}^{*}(t)\right)-\mathbf{y}(t) \\
=\mathbf{H}(q ; \theta)^{-1}\left[\mathbf{G}(q ; \theta) \mathbf{v}\left(\mathbf{U}^{*}(t)\right)-\mathbf{y}(t)\right]
\end{array}
$$

Applying the Least Squares estimate method yields

$$
\hat{\theta}^{L S}\left(\mathbb{Z}_{v}\right)=\arg \min _{\theta} \frac{1}{N} \sum_{t=1}^{N}\left|\hat{\mathbf{y}}\left(t \mid t-1 ; \theta, \mathbf{U}^{*}(t)\right)-\mathbf{y}(t)\right|^{2}
$$

If the Maximum Likelihood estimate is used, an unbiased estimate $\hat{\theta}^{M L}\left(\mathbb{Z}_{v}\right)$ is obtained. Let $f_{\Delta}(\Delta \mathbf{y})$ be a distribution of prediction error $\Delta \mathbf{y}$, the log likelihood function is given by

$$
L\left(\theta ; \mathbb{Z}_{v}\right)=\sum_{t=1}^{N} \log f_{\Delta}\left[\hat{\mathbf{y}}\left(t \mid t-1 ; \theta, \mathbf{U}^{*}(t)\right)-\mathbf{y}(t)\right]
$$

and the Maximum Likelihood estimate is given by

$$
\hat{\theta}^{M L}\left(\mathbb{Z}_{v}\right)=\arg \max _{\theta} L\left(\theta ; \mathbb{Z}_{v}\right)
$$

If the distribution $f_{\Delta}(\Delta \mathbf{y})$ contains unknown parameters, they can be estimated together with $\theta$ in Eq. (20). 
One of the major challenges in system identification is to determine the model structure, in particular, system order and the number of parameters to identify. As more parameters are used, the prediction error $\Delta \mathbf{y}$ may decrease. However, using too many parameters incurs an over-fitting problem, and increases the covariance of parameter estimation error. There is a tradeoff between data fitting accuracy and error covariance, and this can be addressed with the information theoretic method, such as Akaike's Information Criterion (AIC) [11]. Among possible model structures, AIC picks the one that minimizes the overall penalty of prediction error and its covariance due to use of increased number of parameters, $d$ :

$$
\hat{\theta}^{A I C}\left(\mathbb{Z}_{v}\right)=\arg \min _{\theta, d}\left(-2 L\left(\theta ; \mathbb{Z}_{v}\right)+2 d\right)
$$

AIC allows us to compare multiple model structures with a unified metric. There are a few caveats, however [10].

- The Maximum Likelihood estimate requires a sufficiently large number of data points, and so does the AIC estimate.

- The AIC estimate assumes that the true system is involved in the model set under consideration. If not, the second term in Eq. (21), i.e. $2 d$, must be replaced by Takeuchi's bias adjustment term [11].

\section{APPLICATION TO IN VITRO MICROFLUIDIC EC SPROUTING PROCESS}

The above modeling and identification methodology is applied to an Endothelial Cell sprouting process in an in vitro microfluidic chamber. Figure 4 shows a schematic diagram of the chamber. A collagen gel matrix is formed between microfluidic channels A and B. Human Micro-Vascular Endothelial Cells (hMVEC) are seeded on one side of the gel matrix facing Channel B. Fluids containing growth factors and other molecules are delivered to the gel matrix through both channels. The fluid provided to Channel A contains a higher concentration level of VEGF than that of Channel B, so that a uniform gradient of VEGF concentration can be formed across the gel matrix. In response to the gradient of VEGF provided, ECs sprout out and extend towards the higher VEGF concentration. The sprouting process is observed from beneath using a confocal microscope, which can measure 3-dimensional movements of the individual cells. Figure 1 showed an example of the type of confocal microscopy data we obtained: a sprout that has grown from the monolayer over $48 \mathrm{hr}$. We monitor the growth process using the confocal imaging system and obtain position trajectories for the cells involved in the growth process.

As mentioned previously, the migration speed of a tip cell significantly affects the succeeding sprout formation and vessel structure by stalk cells. Therefore, the migration speed of a tip cell is defined to be the output response to regulate:

$$
y=(\text { tip cell speed })
$$

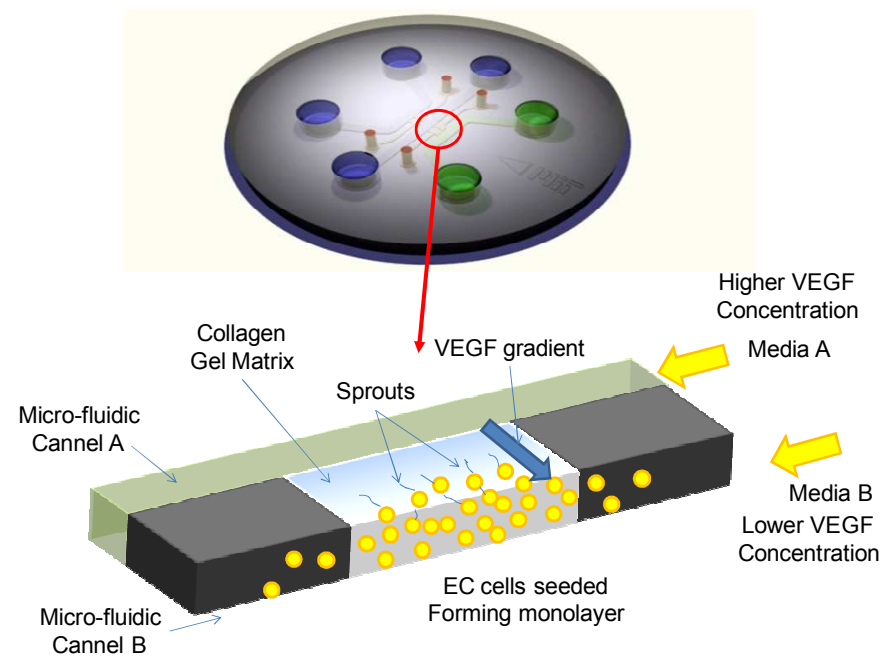

FIGURE 4. IN VITRO MICRO-FLUIDIC DEVICE FOR ANGIOGENIC EC SPROUTING EXPERIMENT [3].

The cues provided to seeded ECs in the in vitro microfluidic environment are many. Over 20 items of basal conditions, ranging from concentration of chemo-attractants to gel matrix stiffness, have been set to their optimal values obtained from a number of experiments. Among these basal conditions, several items are selected for input cues to be manipulated in real time during sprouting experiments. With the double channel microfluidic chamber, both VEGF concentration and its gradient can be varied separately; concentrations of other growth factors, both pro-angiogenic and anti-angiogenic, can be varied. Although these possible input cues are numerous, the principal signals $\mathbf{v}$ with large singular values are just a few. Based on the signaling space reduction method, we can find a particular combination of these input cues with the proportion given by the eigenvector associated with the large singular values.

For simplicity, let us take only the first singular value and use $v_{1}$ as the sole input to the linear dynamical system. Then, the system becomes a single-input, single-output system. Consider an Auto-Regressive with eXogenous input (ARX) model with transfer functions in the following form:

$$
G(q, \theta)=\frac{B(q)}{A(q)} \quad H(q, \theta)=\frac{1}{A(q)}
$$

where $A(q)$ and $B(q)$ are polynomials of orders $n_{a}$ and $n_{b}$ respectively. From Eq.(16) the predictor is given by

$$
\hat{y}(t \mid t-1 ; \theta)=\phi^{T}(t) \theta
$$

where

$$
\begin{aligned}
& \phi(t)=\left[-y(t-1), \ldots,-y\left(t-n_{a}\right), u(t-1), \ldots, u\left(t-n_{b}\right)\right]^{T} \\
& \theta=\left(a_{1}, a_{2}, \ldots, a_{n_{a}}, b_{1}, b_{2}, \ldots, b_{n_{b}}\right)^{T}
\end{aligned}
$$


Given experimental data, an optimal estimate of the parameters can be obtained based on Least Squares (Eq. (18)), Maximum Likelihood (Eq. (20)), or AIC (Eq. (21)).

As of this paper submission day, experimental data usable for this system identification are not available. In particular, measurements of signals for determining the reduced signaling space must be conducted. This experiment only requires offline measurements for identifying the components constituting the most significant principal signal, and is not required for real time control. Once the principal signaling space is identified, the ARX dynamic model can be determined, and the complete Hammerstein model will be obtained.

\section{CONCLUSION}

A Hammerstein-type nonlinear dynamic model and its identification method have been presented. Control of biological systems, such as angiogenic EC sprouting processes, is a challenging task, because of the high degree of complexity and dimensionality. Cue-signal-response processes, which govern cell behaviors, are highly nonlinear and complex, but its signaling space can be reduced to just a few principal axes. This paper exploits this signaling space property, and has proposed to identify and control the otherwise highly complex system with just a few, but informative axes.

There are many open questions to answer. First, the signaling molecules that can be measured with currently available techniques are rather limited. It is a critical question whether the principal signaling space can be identified with the limited signals that are measurable. Second, although the linear portion of system identification can be performed rigorously and systematically, the nonlinear function generation is rather heuristic. Signaling networks available in the literature can be used as a reference and guidelines for constructing a group of building-block nonlinear functions. However, it is a trial-anderror process.

Although numerous challenges exist, modeling and control of biological systems, in particular, multi-cellular systems, will have high impact upon many fields of life science and biological engineering. In vitro angiogenesis, for example, will be a major breakthrough, if functional vascular systems can be created. Tissue engineering and re-generative medicine will benefit directly if blood vessel development process can be controlled or guided. The same technology can be applied to drug discovery and cancer treatment, both of them will become huge industries for the future.

\section{ACKNOWLEDGMENTS}

The author thanks Professors Roger Kamm and Douglas Lauffenburger of MIT for their cross-disciplinary collaboration, which made this work possible. The author also thanks Levi Wood and the d'Arbeloff Lab members for their contributions to experiments.
This material is based on work supported in part by the Emerging Frontiers in Research and Innovation Program of the National Science Foundation under grant number NSF EFRI0735997. The work is also supported by the Singapore-MIT Alliance for Research and Technology, Bio-Systems and Micromechanics IRG.

\section{REFERENCES}

[1] Lodish, H. at al, "Molecular Cell Biology - 6-th edition", chapters 15,16, Freeman, New York, 2008.

[2] Hautaniemi, S, Kharait, S., Iwabu, A., Wells, A., and Lauffenburger, D., 2005 "Modeling of Signal-Response Cascades Using Decision Tree Analysis", Bio-Informatics, 21(9), pp.2027- 2035.

[3] Chung, S., Sudo, R., Mack, P., Wan, C., Vickerman, V., \& Kamm, R., 2009. "Cell Migration into Scaffolds under Coculture Conditions in a Microfluidic Platform", Lab on a Chip, 9(2), pp.269-275.

[4] Datta, S., 2001. "Exploring Relationship in Gene Expressions: A practical least squares approach", Journal of Gene expression, 9, pp.249-255.

[5] Nguyen, D.V., and Roche, D.M., 2002. "Tumor Classification by Partial Least Squares Using Microarray Gene Expression Data", Bioinformatics, 18(1), pp.39-50.

[6] Janes, K., Kelly, J.R., Gaudet, S., Albeck, J.G., Sorger, P.K., and Lauffenburger, D., 2004. "Cue-Signal-Response Analysis of TNF-Induced Apoptosis by Partial Least Square Regression of Dynamic Multivariate Data", Journal of Computational Biology, 11(4), pp.544-561.

[7] Gerhardt, H., 2008. "VEGF and Endothelial Guidance in Angiogenic Sprouting", in C. Ruhrberg (Ed.) VEGF in development, Molecular Biology Intelligence Unit, chap. 6, (pp. 68-78). Springer New York.

[8] Lamalice, L., Le Boeuf, F., and Huot, J., 2007. "Endothelial Cell Migration During Angiogenesis," Circulation research, Journal of the American Heart Association, 100, pp.782-794.

[9] De Smet, F., Segura, I., De Bock, K., Hohensinner, P., and Cameriet, P., 2009. "Mechanisms of Vessel Branching: Fiolopodia on Endothelial Tip Cells Lead the Way", Arteriosclerosis, Thrombosis, and Vascular Biology, Journal of the American Heart Association, 29, pp.639649.

[10]Ljung, Lennart, 1999. "System Identification: Theory for the User, Second Edition”, Prentice-Hall.

[11]Burnham, K.P., and Anderson, D.R., 2002. "Model Selection and Multi-model Inference - A Practical Information-Theoretic Approach, $2^{\text {nd }}$ Edition," Springer. 